\section{RSP}

http://www.rsp.fsp.usp.br/
Revista de Saúde Pública

\title{
Espaços verdes e mortalidade por doenças cardiovasculares no município do Rio de Janeiro
}

\author{
Ismael Henrique da Silveira', Washington Leite Junger"
}

I Universidade do Estado do Rio de Janeiro. Instituto de Medicina Social. Programa de Pós-Graduação em Saúde Coletiva. Rio de Janeiro, RJ, Brasil

" Universidade do Estado do Rio de Janeiro. Instituto de Medicina Social. Departamento de Epidemiologia. Rio de Janeiro, RJ, Brasil

\section{RESUMO}

OBJETIVO: Investigar a associação entre a exposição aos espaços verdes e a mortalidade por doenças isquêmicas do coração e cerebrovasculares, e o papel do nível socioeconômico nessa relação, no município do Rio de Janeiro, Brasil.

MÉTODOS: Estudo ecológico, tendo os setores censitários como unidade de análise. Foram utilizados os dados de óbitos por doenças isquêmicas do coração e cerebrovasculares, entre residentes com idade acima de 30 anos, ocorridos de 2010 a 2012. A exposição ao verde foi estimada por meio do Índice de Vegetação por Diferença Normalizada, baseado em imagens de satélite. As associações entre a exposição aos espaços verdes e a taxas de mortalidade por doenças isquêmicas do coração e cerebrovasculares, padronizadas por sexo e idade, foram analisadas por meio de modelos condicionais autorregressivos, ajustados pelas densidades de vias de tráfego leve e pesado, proxy de poluição, e pela situação socioeconômica, mensurada pelo Índice de Desenvolvimento Social. Também foram realizadas análises estratificadas por níveis socioeconômicos, dados pelos tercis do Índice de Desenvolvimento Social.

RESULTADOS: Entre os setores mais verdes, com Índice de Vegetação por Diferença Normalizada acima do terceiro quartil, a redução da mortalidade por doenças isquêmicas do coração foi de 6,7\% (IC95\% 3,5-9,8) e por cerebrovascular foi de 4,7\% (IC95\% 1,2-8,0). Na análise estratificada, o efeito protetor dos espaços verdes na mortalidade por doenças isquêmicas do coração foi observado entre os setores mais verdes de todos os estratos, sendo maior para os de menor nível socioeconômico (8,6\%; IC95\% 1,8-15,0). No caso da mortalidade por doenças cerebrovasculares, o efeito protetor foi verificado apenas para os setores mais verdes do nível socioeconômico mais baixo (9,6\%; IC95\% 2,3-16,5).

CONCLUSÕES: As taxas de mortalidade por doenças isquêmicas do coração e cerebrovasculares são inversamente associadas à exposição aos espaços verdes, controlando o nível socioeconômico e a poluição do ar. O efeito protetor dos espaços verdes é maior entre os setores de nível socioeconômico mais baixo.

DESCRITORES: Doenças Cardiovasculares, mortalidade. Áreas Verdes. Saúde da População Urbana. Estudos Ecológicos. 


\section{INTRODUÇÃO}

As doenças cardiovasculares (DCV) estão entre os principais problemas mundiais de saúde. De acordo com a Organização Mundial de Saúde (OMS), das 56 milhões de mortes ocorridas no mundo em 2012, mais de 17,5 milhões (31\%) foram atribuídas às DCV, sendo 7,4 milhões (13\%) causadas pelas doenças isquêmicas do coração (DIC) e 6,7 milhões (12\%), pelas doenças cerebrovasculares (DCBV) ${ }^{1}$. No Brasil, em 2013, as DCV foram a causa de 339.672 óbitos, $28 \%$ do total. Foi a segunda causa de morte entre as pessoas com 20 a 59 anos de idade, atrás apenas das causas externas, e a primeira causa entre pessoas com mais de 60 anos $^{\mathrm{a}}$.

Tradicionalmente, os estudos epidemiológicos sobre as DCV têm focado nos fatores de risco comportamentais e biológicos relacionados ao indivíduo ${ }^{2}$. Sua alta carga tem sido atribuída principalmente à elevada prevalência de fatores modificáveis como tabagismo, uso prejudicial de álcool, dieta inadequada e inatividade física, precursores de hipertensão, hiperlipidemia e diabetes ${ }^{1,3,4}$. No entanto, tem havido um crescente interesse, em estudos epidemiológicos, sobre a influência de determinantes contextuais referentes ao local de moradia ${ }^{2,5}$.

Dentre os fatores contextuais ou ambientais que influenciam a saúde, a exposição aos espaços verdes tem sido amplamente pesquisada ${ }^{6}$. Os espaços verdes se referem a espaços com presença de vegetação, como florestas, parques, praças, jardins e ruas arborizadas. Diversos estudos em epidemiologia ambiental têm observado benefícios da exposição aos espaços verdes para a saúde mental, o aumento da prática de atividades físicas e a redução da morbimortalidade geral e por causas específicas, como as $\mathrm{DCV}^{6-8}$.

Apesar das evidências de benefícios dos espaços verdes para a saúde, estudos sobre o tema em países de média e baixa renda são raros, especialmente na região da América Latina, cuja proporção da população urbana já chega a $80 \%$ e tende a continuar aumentando9. Diante desse cenário, investigações sobre as influências de fatores ambientais na saúde da população são importantes para orientar medidas de promoção da saúde no ambiente urbano. O objetivo desse estudo foi investigar a associação entre a exposição aos espaços verdes e a mortalidade por DIC e DCBV, e o papel do nível socioeconômico nessa relação, no município do Rio de Janeiro, Brasil.

\section{MÉTODOS}

Este estudo ecológico foi realizado no município do Rio de Janeiro, entre 2010 e 2012, tendo os setores censitários como unidades de análise. Em 2010, a população do município era de 6.320.446 habitantes, o Índice de Desenvolvimento Humano Municipal era de 0,799, renda per capita de R\$1.492,63 e o Índice de Gini de 0,62, refletindo elevada desigualdade na distribuição de rendab

O território do município é formado por 10.504 setores censitários, sendo 7.922 classificados como normais, 2.212 classificados como aglomerados subnormais, correspondentes às áreas de assentamentos irregulares, e o restante engloba setores sem população ou classificados como hospitais, orfanatos, presídios etc. As análises consideraram apenas os setores normais em função da maior ocorrência de perdas, durante o georreferenciamento, em aglomerados subnormais.

a Ministério da Saúde (BR), Departamento de Informática do SUS - DATASUS. Informações de Saúde (TABNET). Brasília (DF); c2008 [citado 12 jan 2015]. Disponível em: http://www2. datasus.gov.br/DATASUS/index.php b Programa das Nações Unidas para o Desenvolvimento. Atlas do Desenvolvimento Humano no Brasil. Brasília (DF); PNUD; c2014 [citado 4 abr 2017]. Disponível em: http://www. atlasbrasil.org.br/2013/pt/

Os dados de mortalidade foram obtidos do Sistema de Informações sobre Mortalidade (SIM) da Secretaria Municipal de Saúde do Rio de Janeiro. Foram considerados os óbitos de pessoas com idade acima de 30 anos e que tiveram como causas básicas as DIC e as DCBV, agrupadas no capítulo IX da 10ª Revisão da Classificação Internacional de Doenças (CID-10), respectivamente entre os códigos I20 a I25 e I60 a I69.

Os endereços dos óbitos foram georreferenciados em três etapas: a padronização dos endereços, o georreferenciamento e a intervenção manual. As duas primeiras etapas foram programadas em R. Na padronização, os erros e abreviações mais frequentes foram substituídos. Na etapa de georreferenciamento, as coordenadas geográficas dos endereços foram obtidas da base 
de endereços do Google Maps, por meio da Interface de Programação de Aplicativos (API) do Google, um conjunto de funções pré-programadas como a de georreferenciamento. Por fim, a intervenção manual foi realizada para se localizar os endereços não encontrados inicialmente.

Após georreferenciados, os óbitos foram agregados por setor censitário. A base cartográfica de setores censitários, relativa ao censo de 2010, foi obtida do Instituto Brasileiro de Geografia e Estatística (IBGE). As razões de mortalidade por DIC e DCBV padronizadas (RMP), por sexo e faixa etária, foram calculadas dividindo-se o número de óbitos observados pelo número de óbitos esperados em cada unidade de análise. Os valores esperados foram calculados com base nos coeficientes de mortalidade do município, pelas respectivas causas e período, por sexo e faixa etária.

A exposição aos espaços verdes foi medida utilizando-se o Índice de Vegetação por Diferença Normalizada (NDVI - Normalized Difference Vegetation Index). O NDVI é constituído a partir de imagens de satélite, servindo para caracterizar a densidade da vegetação de uma região. Os pigmentos clorofilados, presentes nas plantas, produzem um padrão de baixa refletância do comprimento de onda vermelho (VER) e alta refletância próximo do infravermelho (PIV). $\mathrm{O}$ índice é determinado pela equação:

$$
\text { NDVI }=(\text { PIV }- \text { VER }) /(\text { PIV + VER })
$$

O NDVI pode variar entre - 1 e 1. Valores negativos indicam a presença de gelo, água e nuvens; valores em torno de -0,1 e 0,1 correspondem às superfícies descobertas; e valores próximos a 0,7 se referem à vegetação densac .

As imagens foram obtidas da coleção Landsat 4-5TM, do Serviço Geológico dos Estados Unidos (USGS), com resolução de 30 metros. Foram utilizadas apenas aquelas com menos de $10 \%$ de nuvens geradas no período, totalizando sete imagens. Primeiro, foi calculado o NDVI médio do período e, então, calculou-se o NDVI médio de cada setor censitário considerando buffers de 100 metros a partir das suas bordas. Os buffers foram utilizados para que pudéssemos considerar a exposição ao redor do setor e não apenas a encerrada em seu limite.

As densidades de vias de tráfico veicular foram utilizadas como medidas indiretas da poluição do ar, conforme outros estudos epidemiológicos ${ }^{10,11}$. As vias foram separadas em dois grupos de tráfego: leve e pesado. As vias de tráfego leve são aquelas classificadas como locais e coletoras, responsáveis pela distribuição do tráfego interno aos bairros. As vias de tráfego pesado, classificadas como estruturais, arteriais primárias e arteriais secundárias, estabelecem conexões rápidas, atendem deslocamentos distantes e são utilizadas por um elevado volume de veículos. A densidade foi obtida pelo quociente entre o comprimento das vias e a área que inclui o setor censitário e um buffer de 100 metros a partir de suas bordas. A base cartográfica digital de logradouros foi obtida do Instituto Pereira Passos da Prefeitura Municipal do Rio de Janeiro.

O nível socioeconômico foi medido por meio do Índice de Desenvolvimento Social (IDS), que expressa o grau de desenvolvimento de determinada área ${ }^{12}$. É constituído por oito indicadores, com base em dados do Censo de 2010, a saber: proporção de domicílios com abastecimento de água via rede geral, proporção de domicílios com coleta de esgoto via rede geral ou pluvial, proporção de domicílios com coleta de lixo, número médio de banheiros por pessoa, proporção de analfabetismo entre pessoas com idade de 10 a 14 anos, rendimento médio das pessoas responsáveis pelo domicílio (em salários mínimos), proporção de pessoas responsáveis pelo domicílio com rendimento de até dois salários mínimos e proporção de pessoas responsáveis pelo domicílio com rendimento igual ou superior a 10 salários mínimos.

${ }^{c}$ Weier J, Herring D. Measuring Vegetation (NDVI \& EVI). Greenbelt (MD): NASA Earth Observatory; 2000 [citado 30 jun 2016]. Disponível em: https://earthobservatory.nasa.gov/ Features/MeasuringVegetation/ printall.php
Para o cálculo do índice, os indicadores foram primeiramente normalizados, de modo que se tornassem compatíveis, variando entre zero e um. Posteriormente, foi calculada a média aritmética desses indicadores, que corresponde ao valor do IDS.

Foi criada uma variável binária para indicar os setores censitários localizados na costa litorânea. Esses setores são, geralmente, áreas com melhores condições socioeconômicas e presença de vegetação; mas, devido à faixa de areia, tem o valor do NDVI médio reduzido. 
As associações entre os espaços verdes e as RMP foram analisadas por meio de modelos condicionais autorregressivos (CAR - conditional autoregressive) com distribuição gaussiana. As variáveis de desfecho analisadas nos modelos foram o log das RMP por DIC e DCBV para se aproximar de uma distribuição gaussiana. Os modelos foram ajustados por IDS, densidade de vias de tráfego leve, densidade de vias de tráfego pesado, o indicador de setor litorâneo e a média do NDVI (categorizada em quartis). O valor do risco relativo pode ser obtido pelo exponencial dos coeficientes da regressão.

As análises foram realizadas em duas etapas: considerando todos os setores incluídos no estudo e estratificando por tercis de IDS (para investigarmos a modificação de efeito do nível socioeconômico). Os estratos foram classificados em: baixo (IDS de 0,264 a 0,586); intermediário (IDS de 0,586 a 0,642); e alto (IDS de 0,642 a 0,900). A autocorrelação espacial foi avaliada por meio do Índice de Moran I dos resíduos da regressão linear multivariada. Os modelos com dependência espacial foram mantidos em todas as análises para evitar a subestimação da variância dos estimadores dos coeficientes das regressões.

O modelo CAR é utilizado em situações em que está presente a autocorrelação espacial. Ele é especificado para um conjunto de distribuições de probabilidade condicionais de cada observação, considerando os valores das demais. Assumindo que $\{\mathrm{Y}(\mathrm{si}):$ si $€$ (s1... sn) $\}$ seja um processo gaussiano em que \{s1... sn\} forma o conjunto de áreas S, o modelo pode ser definido, especificando a média e a variância, conforme as equações ${ }^{13}$ :

$$
\begin{gathered}
\mathrm{E}\left[\mathrm{Y}\left(\mathrm{s}_{\mathrm{i}}\right) \mid \mathrm{Y}\left(\mathrm{s}_{(-\mathrm{i})}\right)\right]=\mathrm{x}\left(\mathrm{s}_{\mathrm{i}}\right)^{\prime} \beta+\sum_{\mathrm{j}=\mathrm{i}}^{\mathrm{n}} \mathrm{c}_{\mathrm{ij}}\left[\mathrm{Y}\left(\mathrm{s}_{\mathrm{j}}\right)-\mathrm{x}\left(\mathrm{s}_{\mathrm{i}}\right)^{\prime} \beta,\right. \\
\operatorname{Var}\left[\mathrm{Y}\left(\mathrm{s}_{\mathrm{i}}\right) \mid \mathrm{Y}\left(\mathrm{s}_{(-\mathrm{i})}\right)\right]=\sigma_{\mathrm{i}}^{2}, \text { sendo } \mathrm{i}=1, \ldots, \mathrm{N},
\end{gathered}
$$

Em que $\mathrm{Y}\left(\mathrm{s}_{(-\mathrm{i})}\right)=\left\{\mathrm{Y}\left(\mathrm{s}_{\mathrm{j}}\right): \mathrm{j} \neq \mathrm{i}\right\}, \mathrm{Y}\left(\mathrm{s}_{\mathrm{i}}\right)=\mu_{\mathrm{i}} \mu_{\mathrm{i}}$ nesse caso é o log da RMP, $\operatorname{Var}\left[\mathrm{Y}\left(\mathrm{s}_{\mathrm{i}}\right) \mid \mathrm{Y}\left(\mathrm{s}\left(\mathrm{s}_{\mathrm{i}}\right)\right)\right]$ é a variância condicional, $c_{i j}$ denotam os parâmetros de dependência espacial, em particular $c_{i i}=0, c_{i j}$ é diferente de zero quando s é vizinha de $\mathrm{s}_{\mathrm{i}}$. As matrizes $\mathrm{C}=\left(\mathrm{c}_{\mathrm{ij}}\right)$ e $\Sigma_{\mathrm{c}}=\operatorname{diag}\left[\sigma_{1}^{2} \sigma_{2}^{2}, \ldots, \sigma_{\mathrm{n}}^{2}\right]$ são obtidas e $\operatorname{assim} \Sigma_{\mathrm{Y}}=(\mathrm{I}-\mathrm{C})^{-1} \Sigma_{\mathrm{c}}$.

A estrutura de $C$ é usualmente especificada com um único parâmetro baseado na matriz de vizinhança definida, o W. C é dado por $\rho_{c} W$, em que $\rho_{c}$ é o parâmetro de dependência espacial a ser estimado. Os parâmetros são estimados pelo método de máxima verossimilhança.

Também foram realizadas análises descritivas, por meio de mapas de distribuição das RMP por DIC e DCBV, do IDS e do NDVI por setores censitários. Como alguns setores censitários possuem populações muito pequenas, acarretando instabilidade para as taxas brutas, as RMP foram suavizadas pelo método Bayesiano Empírico Global, cuja matriz de vizinhança foi definida por contiguidade.

As análises estatísticas e o georreferenciamento foram realizados no R 3.1.1 e os procedimentos de geoprocessamento foram realizados no programa QGIS 2.6.1. O estudo foi aprovado pelos Comitês de Ética em Pesquisa do Instituto de Medicina Social da Universidade do Estado do Rio de Janeiro e da Secretaria Municipal de Saúde do Rio de Janeiro (Parecer 531635).

\section{RESULTADOS}

Foram notificados 25.959 óbitos por DIC ou DCBV, entre pessoas com idade acima de 30 anos, entre 2010 e 2012, residentes no município do Rio de Janeiro. Puderam ser georreferenciados 23.800 óbitos, resultando em 9,2\% de perdas. Os óbitos localizados em setores normais, incluídos no estudo, totalizaram 21.689, 11.817 por DIC e 9.872 por DCBV.

A Tabela 1 apresenta o resumo das estatísticas descritivas das variáveis incluídas no estudo. Os mapas de distribuição das RMP por DIC e DCBV, do IDS e do NDVI médio por setores censitários no município do Rio de Janeiro, estão apresentados na Figura (A-D). 
As distribuições espaciais das RMP por DIC (Figura, A) e DCBV (Figura, B), não exibiram nenhum padrão definido. A distribuição do IDS (Figura, $C$ ) mostrou maior concentração de valores baixos entre as zonas norte e oeste da cidade. Os valores elevados se concentraram na região Sul. O mapa do NDVI médio (Figura, D) evidenciou a concentração de valores baixos na zona norte da cidade. Os setores mais verdes se concentraram entre as zonas sul e oeste; esta última é a região que possui maior quantidade de áreas menos urbanizadas, como sítios e chácaras.

Tabela 1. Estatísticas descritivas das variáveis incluídas nas análises, referentes aos setores censitários normais do município do Rio de Janeiro.

\begin{tabular}{lcccccc}
\hline Variável & Mínimo & $\mathbf{1}^{\mathbf{0}}$ quartil & Mediana & Média & $3^{\mathbf{0}}$ quartil & Máximo \\
\hline RMP por doenças isquêmicas do coração & 0,000 & 0,000 & 0,707 & 0,963 & 1,400 & 23,030 \\
RMP por doenças cerebrovasculares & 0,000 & 0,000 & 0,648 & 0,978 & 1,393 & 29,950 \\
Índice de Desenvolvimento Social (IDS) & 0,265 & 0,575 & 0,608 & 0,623 & 0,675 & 0,900 \\
Densidade de vias leves $\left(\mathrm{m}^{-1}\right)$ & 0,0000 & 0,0100 & 0,0136 & 0,0139 & 0,0174 & 0,0472 \\
Densidade de vias pesadas $\left(\mathrm{m}^{-1}\right)$ & 0,0000 & 0,0000 & 0,0000 & 0,0023 & 0,0038 & 0,0276 \\
Média do Índice de Vegetação por Diferença Normalizada & & & & & \\
$\quad$ Todos setores normais & $-0,097$ & 0,045 & 0,086 & 0,111 & 0,153 & 0,593 \\
$\quad$ Setores com IDS baixo & $-0,044$ & 0,061 & 0,061 & 0,140 & 0,192 & 0,576 \\
$\quad$ Setores com IDS elevado & $-0,061$ & 0,039 & 0,070 & 0,087 & 0,120 & 0,580 \\
$\quad$ Setores com IDS alto & $-0,097$ & 0,041 & 0,085 & 0,105 & 0,154 & 0,593 \\
\hline
\end{tabular}

RMP: razão de mortalidade padronizada por faixa etária e gênero
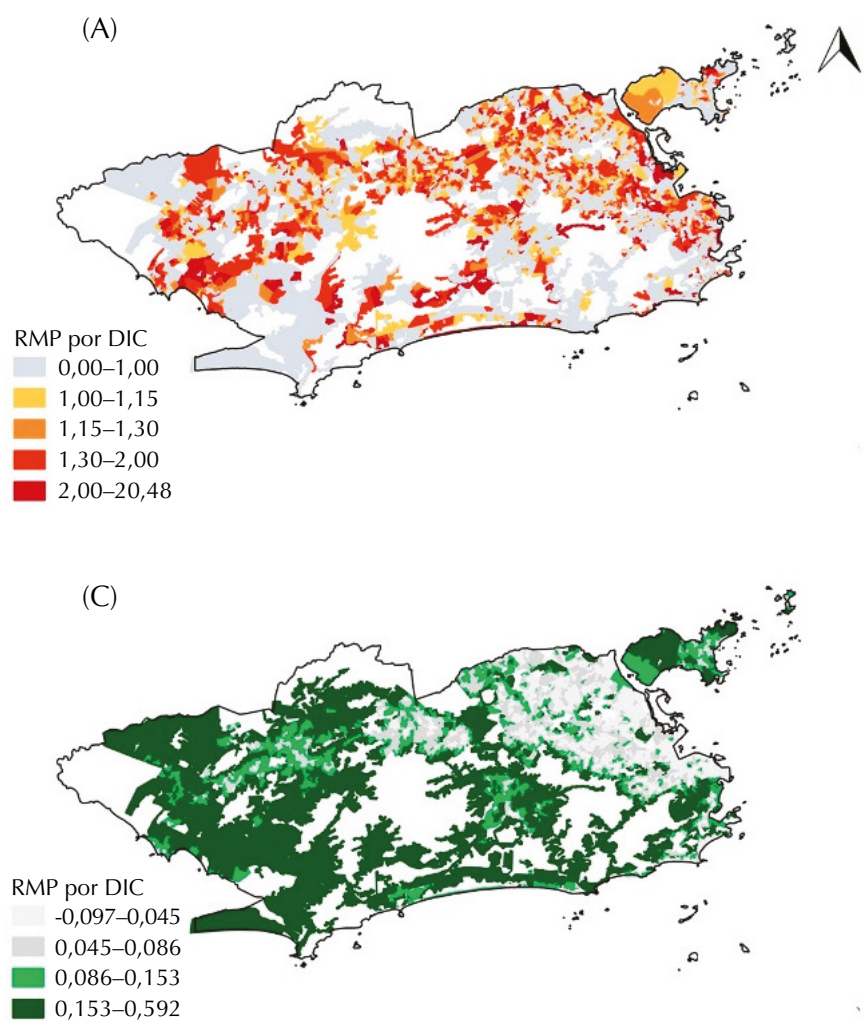
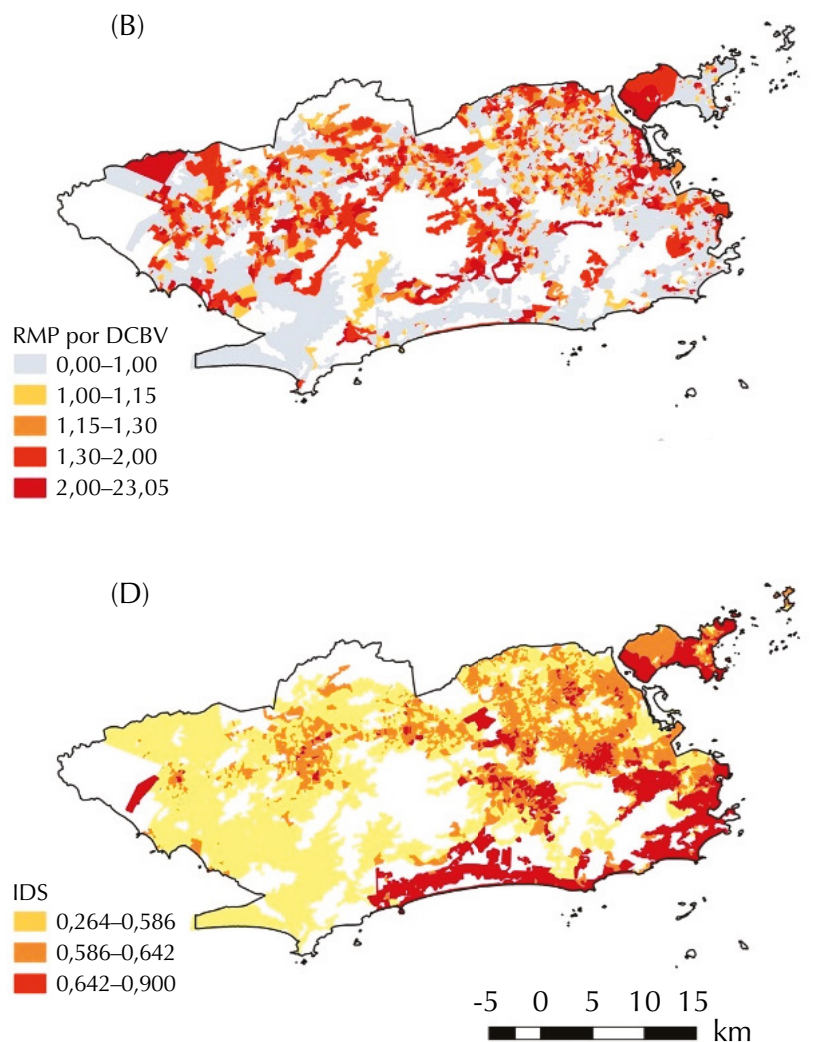

RMP: razão de mortalidade padronizada por faixa etária e gênero

Figura. (A) Distribuição espacial da razão de mortalidade por doenças isquêmicas do coração (DIC), por setores censitários normais, no município do Rio de Janeiro, de 2010 a 2012; (B) Distribuição espacial da razão de mortalidade por doenças cerebrovasculares (DCBV), por setores censitários normais, no município do Rio de Janeiro, de 2010 a 2012; (C) Distribuição espacial do Índice de Desenvolvimento Social (IDS), por setores censitários normais, no município do Rio de Janeiro em 2010; (D) Distribuição espacial do Índice de Vegetação por Diferença Normalizada (NDVI) médio, por setores censitários, no município do Rio de Janeiro, de 2010 a 2012. 
As análises multivariadas (Tabela 2), ajustadas pelo IDS, densidades de vias leves e pesadas e pelo indicador de setores costeiros, mostraram o efeito protetor dos espaços verdes na mortalidade por DIC nos setores com NDVI acima da mediana. Para a mortalidade por DCBV, o efeito protetor foi observado apenas nos setores com NDVI acima do terceiro quartil. Entre esses setores mais verdes, com NDVI acima do terceiro quartil, a redução da mortalidade por DIC foi de 6,7\% (IC95\% 3,5-9,8) e por DCBV foi de 4,7\% (IC95\% 1,2-8,0). As RMP também foram associadas inversamente ao IDS e diretamente à exposição à poluição do ar. As autocorrelações dos modelos, dadas pelo coeficiente $\rho$, foram positivas, embora tenham sido próximas de zero: $0,085(0,051 ; 0,119)$ para o modelo das DIC e $0,078(0,004 ; 0,151)$ para o modelo das DCBV.

Quanto aos modelos estratificados por tercis do IDS (Tabela 3), foi observado o efeito protetor dos espaços verdes na mortalidade por DIC entre os setores mais verdes (NDVI acima do terceiro quartil) em todos os estratos. Esse efeito protetor foi maior para os setores com o IDS baixo. Entre os setores mais verdes, a redução da mortalidade foi de 8,6\% (IC95\% 1,8-15.0) no estrato com IDS baixo, 6,3\% (IC95\% 1,1-11.2) no estrato intermediário e 6,7\% (IC95\% 2,0-11,2) no mais elevado. Para a mortalidade por DCBV, o efeito protetor da exposição foi verificado apenas para os setores mais verdes do estrato mais baixo do IDS. Nessas áreas, a redução da mortalidade foi de 9,6\% (IC95\% 2,3-16,5). Os coeficientes de autocorrelação espacial significativos entre os modelos com a mortalidade por DIC foram 0,093 (0,002; 0,184) para os setores com IDS baixo; e -0,073 (-0,143; $-0,002)$ para os setores com IDS alto. Entre os modelos com a mortalidade por DCBV, apenas os setores com IDS alto tiveram autocorrelação significativa e igual a 0,171 $(0,104 ; 0,239)$.

Tabela 2. Coeficientes $(\beta)$ dos modelos condicionais autorregressivos para a associação entre o $\log$ das RMP, por DIC e DCBV, e o NDVI médio, no município do Rio de Janeiro, 2010 a 2012.

\begin{tabular}{|c|c|c|c|c|}
\hline \multirow{2}{*}{ Variável } & \multicolumn{2}{|c|}{ DIC } & \multicolumn{2}{|c|}{ DCBV } \\
\hline & $\beta$ & IC95\% & $\beta$ & IC95\% \\
\hline Índice de Desenvolvimento Social & $-0,705$ & $-0,812--0,599$ & $-1,413$ & $-1,569--1,257$ \\
\hline Indicador de setores costeiros & 0,014 & $-0,135-0,164$ & 0,017 & $-0,084-0,119$ \\
\hline Densidade de vias leves & 1,958 & $1,861-2,056$ & 3,328 & $0,993-5,663$ \\
\hline Densidade de vias pesadas & 4,606 & $2,369-6,843$ & 4,798 & $0,753-8,843$ \\
\hline \multicolumn{5}{|l|}{ Quartos do NDVI médio } \\
\hline Q2 & 0,023 & $-0,008-0,054$ & 0,010 & $-0,022-0,043$ \\
\hline Q3 & $-0,043$ & $-0,075--0,012$ & $-0,011$ & $-0,044-0,022$ \\
\hline Q4 & $-0,069$ & $-0,101--0,038$ & $-0,048$ & $-0,083--0,012$ \\
\hline
\end{tabular}

RMP: razão de mortalidade padronizada por faixa etária e gênero; DIC: doenças isquêmicas do coração; DCBV: doenças cerebrovasculares; NDVI: Normalized Difference Vegetation Index (Índice de Vegetação por Diferença Normalizada)

Tabela 3. Coeficientes ( $\beta$ ) dos modelos condicionais autorregressivos para as associações entre o log das RMP, por DIC e DCBV, e o NDVI médio, no município do Rio de Janeiro, 2010 a 2012, estratificados por tercis do IDS.

\begin{tabular}{|c|c|c|c|c|}
\hline \multirow{2}{*}{\multicolumn{2}{|c|}{ Quartos do NDVI médio }} & \multirow{2}{*}{$\begin{array}{c}1^{a} \text { estrato do IDS }{ }^{a} \\
\beta(\text { IC } 95 \%)\end{array}$} & \multirow{2}{*}{$\begin{array}{c}2^{\circ} \text { estrato do IDS } \\
\beta(\text { IC95 } \%) \\
\end{array}$} & \multirow{2}{*}{$\begin{array}{c}3^{\circ} \text { estrato do IDS } \\
\beta(\text { IC95 } \%) \\
\end{array}$} \\
\hline & & & & \\
\hline \multirow[t]{3}{*}{ DIC } & Q2 & $\begin{array}{c}-0,043 \\
(-0,103-0,017)\end{array}$ & $\begin{array}{c}0,026 \\
(-0,025-0,077)\end{array}$ & $\begin{array}{c}0,045 \\
(-0,003-0,093)\end{array}$ \\
\hline & Q3 & $\begin{array}{c}-0,044 \\
(-0,105-0,018)\end{array}$ & $\begin{array}{c}-0,034 \\
(-0,085-0,018)\end{array}$ & $\begin{array}{c}-0,040 \\
(-0,089-0,009)\end{array}$ \\
\hline & Q4 & $\begin{array}{c}-0,090 \\
(-0,163--0,018)\end{array}$ & $\begin{array}{c}-0,065 \\
(-0,119--0,011)\end{array}$ & $\begin{array}{c}-0,069 \\
(-0,119--0,020)\end{array}$ \\
\hline \multirow[t]{3}{*}{ DCBV } & Q2 & $\begin{array}{c}0,003 \\
(-0,062-0,068)\end{array}$ & $\begin{array}{c}0,010 \\
(-0,045-0,065)\end{array}$ & $\begin{array}{c}-0,001 \\
(-0,046-0,045)\end{array}$ \\
\hline & Q3 & $\begin{array}{c}-0,011 \\
(-0,078-0,055)\end{array}$ & $\begin{array}{c}0,005 \\
(-0,050-0,060)\end{array}$ & $\begin{array}{c}-0,031 \\
(-0,078-0,015\end{array}$ \\
\hline & Q4 & $\begin{array}{c}-0,101 \\
(-0,180--0,023)\end{array}$ & $\begin{array}{c}-0,020 \\
(-0,077-0,038)\end{array}$ & $\begin{array}{c}-0,036 \\
(-0,083-0,012)\end{array}$ \\
\hline
\end{tabular}

RMP: razão de mortalidade padronizada por faixa etária e gênero; DIC: doenças isquêmicas do coração; DCBV: doenças cerebrovasculares; IDS: Índice de Desenvolvimento Social; NDVI: Normalized Difference Vegetation Index (Índice de Vegetação por Diferença Normalizada)

a Ajustado pela densidade de vias de tráfego leve e pesado.

${ }^{\text {b }}$ Ajustado pelo indicador de setores costeiros, pela densidade de vias de tráfego leve e pesado. 


\section{DISCUSSÃO}

Os resultados deste estudo evidenciaram o efeito protetor dos espaços verdes na mortalidade por DIC e DCBV no município do Rio de Janeiro, além do efeito protetor do nível socioeconômico e do efeito deletério da poluição do ar. Os riscos de mortalidade foram menores entre os setores mais verdes, com NDVI médio que varia entre 0,11 e 0,59.

O município do Rio de Janeiro possui arborização em torno de $72 \%$ dos domicílios, ocupando a $12^{\text {a }}$ posição entre as capitais brasileiras (Campo Grande é a primeira, com 96,5\%; São Paulo é a $10^{\mathrm{a}}$, com $75 \%$; Manaus, Belém e Rio Branco ocupam as posições $25^{\mathrm{a}}, 26^{\mathrm{a}}$ e $27^{\mathrm{a}}$, com $24 \%$, $22,3 \%$ e $14 \%$ respectivamente) $)^{d}$. A distribuição do NDVI na cidade do Rio de Janeiro é bastante desigual, sendo a zona norte a que possui os menores índices. No entanto, o município possui um recente plano diretor de arborização, que visa nortear ações de ampliação de arborização , e iniciativas como o Imposto Predial e Territorial Urbano (IPTU) verde e os viveiros municipais de mudas estão em vias de ser implantadas.

Os resultados são consistentes com outros estudos. Em uma investigação ecológica, em dois municípios nos EUA, foi observado o menor risco de mortalidade por AVC em áreas com menor poluição, maior renda e maior NDVI ${ }^{14}$. Em um estudo transversal, realizado na Nova Zelândia, o risco da ocorrência de DCV foi menor entre residentes de regiões com maior proporção de espaços verdes ${ }^{15}$. Essa associação se manteve mesmo após o ajuste pelo nível de atividade física. Um estudo longitudinal, realizado em Ontario, Canadá, verificou que residentes de locais com maior NDVI apresentaram menores taxas de mortalidade por DCV, dentre outros desfechos ${ }^{16}$. A relação continuou significativa mesmo após o ajuste por poluição atmosférica. Um estudo realizado em São Paulo observou a associação inversa entre o total de área verde por metro quadrado e o risco de DCV entre idosos, independentemente de fatores socioeconômicos e da renda média da região de moradia ${ }^{17}$.

A relação entre os espaços verdes e a saúde cardiovascular pode ser mediada pela prática de atividades físicas, pela melhoria das condições psicossociais, como redução do estresse e aumento do contato e da coesão social, além da melhoria da qualidade do ar e do conforto térmico ${ }^{6,7}$.

A prática de atividades físicas é, supostamente, uma das principais vias pela qual se dão os benefícios dos espaços verdes na saúde. A disponibilidade de espaços como parques, jardins e ruas arborizadas, tem o potencial de promover a atividade física, que está associada à redução de inúmeros precursores das $\mathrm{DCV}^{18,19}$. Já os benefícios sobre as condições psicossociais provêm do contato com o espaço natural. Esse contato repercute em efeitos positivos na saúde mental, como nos níveis de estresse e ansiedade, na sensação de bemestar e aumento da interação social, por meio do envolvimento em atividades físicas e sociais $^{20,21}$. As condições psicossociais, por sua vez, podem afetar a saúde cardiovascular via mecanismos patofisiológicos e comportamentais ${ }^{5}$.

Foram também observadas relações inversas entre a mortalidade por DIC e por DCBV e a situação socioeconômica, medida pelo IDS. A morbimortalidade cardiovascular tem sido associada à situação socioeconômica tanto individual, quanto referente ao local de moradia ${ }^{22}$. $\mathrm{Na}$ análise estratificada por tercis do IDS, o efeito protetor dos espaços verdes foi maior para

${ }^{\mathrm{d}}$ Instituto Brasileiro de Geografia e Estatística. Censo Demográfico 2010: características urbanísticas do entorno dos domicílios. Rio de Janeiro: IBGE; 2010 [citado 31 ago 2017]. Disponível em: http://www.ibge.gov.br/home/ estatistica/populacao/censo2010/ entorno/default_entorno.shtm

e Prefeitura do Rio de Janeiro, Fundação Parques e Jardins. Plano diretor de Arborização Urbana da Cidade do Rio de Janeiro. Rio de Janeiro: FP]; 2015 [citado 31 ago 2017]. Disponível em: http://www. rio.rj.gov.br/web/fpj/plano-diretorde-arborizacao-urbana o menor nível socioeconômico no caso da mortalidade por DIC, e observado apenas para o mesmo estrato no caso da mortalidade por DCBV. Esses resultados podem estar indicando a importância da exposição ao verde entre a população em desvantagem socioeconômica. De acordo com Mitchell e Popham ${ }^{23}$ a exposição tem o potencial de reduzir as iniquidades em saúde, visto que a diferença de mortalidade entre níveis socioeconômicos extremos foi menor em locais mais verdes.

O efeito protetor dos espaços verdes também podem se dar por meio da redução da poluição atmosférica e da temperatura ${ }^{6}$. No entanto, como a poluição do ar foi ajustada neste estudo, é provável que os efeitos da exposição se dessem, sobretudo, por outras vias. De todo modo, as associações dos indicadores de poluição atmosférica com a mortalidade por DIC e por DCBV foram positivas, em conformidade com resultados de outros estudos ${ }^{24}$. 
Uma das limitações deste estudo é a exclusão dos setores censitários classificados como aglomerados subnormais, referentes às áreas de assentamentos irregulares, onde vivem $22 \%$ da população. A opção se deu pela maior ocorrência de perdas durante o georreferenciamento, em decorrência principalmente da infraestrutura urbana dessas localidades. Tais perdas acarretam a subestimação das taxas de mortalidade nessas áreas. Mesmo na análise considerando todos os setores, não mostrada no resultado, a redução do risco de mortalidade por DIC e DCBV foi observada entre os setores censitários mais verdes, embora tenha sido menor.

Dentre outras limitações, está a indisponibilidade de informações sobre a prevalência de fatores de risco para as DCV, em nível de setor censitário, como tabagismo, hábitos alimentares, hipertensão, diabetes e dislipidemia. Porém, de acordo com Diez-Roux ${ }^{25}$, a prevalência desses fatores tem sido relacionada a características socioeconômicas do local de moradia, mensuradas a partir de informações censitárias. Nesse sentido, a condição socioeconômica, representada no presente estudo pelo IDS, poderia estar agindo como um marcador desses determinantes.

A medida da exposição, dada pelo NDVI médio, se refere ao período em que ocorreram os óbitos, desconsiderando variações ao longo da vida ou do dia, devido a migrações e às diferenças de exposição entre os locais de moradia e de trabalho. Outros aspectos relacionados aos espaços verdes que podem afetar seu uso também não foram considerados, como acessibilidade, oportunidades de atividades, qualidade dos atributos físicos, aspectos estéticos e segurança ${ }^{7,18}$.

Apesar das limitações, o presente estudo corrobora a importância de fatores contextuais para a saúde cardiovascular. Especialmente ressalta o efeito protetor dos espaços verdes na mortalidade por DIC e DCBV, sobretudo nas áreas mais carentes. Até então, são poucas as investigações dessa natureza conduzidas em países de média e baixa renda, especialmente na América Latina. Estudos futuros, com desenhos mais refinados que avancem na investigação dos mecanismos e mediadores dos efeitos de fatores contextuais são fundamentais para o melhor entendimento dessas relações, bem como o efeito dos diferentes tipos e características dos espaços verdes. Essas informações são de grande relevância na orientação de medidas de promoção da saúde no espaço urbano, voltadas para o controle das diversas doenças crônicas, focadas tanto em aspectos individuais quanto contextuais.

\section{REFERÊNCIAS}

1. World Health Organization. Global status report on noncommunicable diseases 2014. Geneva: WHO; 2014 [citado 5 jul 2015].

Disponível em: http://www.who.int/nmh/publications/ncd-status-report-2014/en/

2. Diez-Roux AV, Mujahid MS, Hirsch JA, Moore K, Moore LV. The Impact of Nneighborhoods on CV risk. Glob Heart. 2016;11(3):353-63. https://doi.org/10.1016/j.gheart.2016.08.002

3. Arboix A. Cardiovascular risk factors for acute stroke: risk profiles in the different subtypes of ischemic stroke. World J Clin Cases. 2015;3(5):418-29. https://doi.org/10.12998/wjcc.v3.i5.418

4. Prasad DS, Kabir Z, Dash AK, Das BC. Cardiovascular risk factors in developing countries: a review of clinico-epidemiological evidence. Eur J Cancer. 2010;5(4):115-23. https://doi.org/10.1016/j.cvdpc.2010.09.001

5. Neylon A, Canniffe C, Anand S, Kreatsoulas C, Blake GJ, Sugrue D, et al. A global perspective on psychosocial risk factors for cardiovascular disease. Prog Cardiovasc Dis. 2013;55(6):574-81. https://doi.org/10.1016/j.pcad.2013.03.009

6. Nieuwenhuijsen MJ, Khreis H, Triguero-Mas M, Gascon M, Dadvand P. Fifty Shades of Green: pathway to healthy urban living. Epidemiology. 2017;28(1):63-71. https//doi.org/10.1097/EDE.0000000000000549

7. Lee AC, Maheswaran R. The health benefits of urban green spaces: a review of the evidence. J Public Health (Oxf). 2011;33(2):212-22. https://doi.org/10.1093/pubmed/fdq068

8. Berg M, Wendel-Vos W, Poppel M, Kemper H, Mechelen W, Maas J. Health benefits of green spaces in the living environment: a systematic review of epidemiological studies. Urban For Urban Green. 2015;14(4):806-16. https://doi.org/10.1016/j.ufug.2015.07.008 
9. United Nations, Department of Economic and Social Affairs, Population Division. World urbanization prospects: the 2014 revision. New York; 2014 [citado 7 jul 2015]. Disponível em: https://esa.un.org/unpd/wup/publications/files/wup2014-highlights.Pdf

10. Kan H, Heiss G, Rose KM, Whitsel EA, Lurmann F, London SJ. Prospective analysis of traffic exposure as a risk factor for incident coronary heart disease: the Atherosclerosis Risk in Communities (ARIC) study. Environ Health Perspect. 2008;116(11):1463-8. https://doi.org/10.1289/ehp.11290

11. Habermann M, Gouveia N. Tráfego veicular e mortalidade por doenças do aparelho circulatório em homens adultos. Rev Saude Publica. 2012;46(1):26-33. https://doi.org/10.1590/S0034-89102011005000079

12. Cavallieri F, Lopes GP. Índice de Desenvolvimento Social (IDS) da Cidade do Rio de Janeiro (2000-2010). Rio de Janeiro: Instituto Pereira Passos (IPP); 2014 [citado 4 abr 2014]. Disponível em: http://portalgeo.rio.rj.gov.br/estudoscariocas/download/2394_\%C3\%8Dndice\%20de\%20 Desenvolvimento\%20Social_IDS.pdf

13. Waller LA, Gotway CA. Applied spatial statistics for public health data. Hoboken (NJ): John Wiley \& Sons; 2004. (Wiley Series on Probability and Statistics, 368).

14. Hu Z, Liebens J, Rao KR. Linking stroke mortality with air pollution, income, and greenness in northwest Florida: an ecological geographical study. Int J Health Geogr. 2008;7:20. https://doi.org/10.1186/1476-072X-7-20

15. Richardson EA, Pearce J, Mitchell R, Kingham S. Role of physical activity in the relationship between urban green space and health. Public Health. 2013;127(4):318-24. https://doi.org/10.1016/j.puhe.2013.01.004

16. Villeneuve PJ, Jerrett M, Su JG, Burnett RT, Chen H, Wheeler AJ, et al. A cohort study relating urban green space with mortality in Ontario, Canada. Environ Res. 2012;115:51-8. https://doi.org/10.1016/j.envres.2012.03.003

17. Massa KHC, Pabayo R, Lebrão ML, Chiavegatto Filho ADP. Environmental factors and cardiovascular diseases: the association of income inequality and green spaces in elderly residents of São Paulo, Brazil. BMJ Open. 2016;6(9):e011850. https|://doi.org/10.1136/bmjopen-2016-011850

18. Kaczynski AT, Henderson KA. Environmental correlates of physical activity: a review of evidence about parks and recreation. Leis Sci. 2007;29(4):315-54. https://doi.org/10.1080/01490400701394865

19. Shanahan DF, Franco L, Lin BB, Gaston KJ, Fuller RA. The benefits of natural environments for physical activity. Sport Med. 2016;46(7):989-95. https://doi.org/10.1007/s40279-016-0502-4

20. Roe JJ, Thompson CW, Aspinall PA, Brewer MJ, Duff EI, Miller D, et al. Green space and stress: evidence from cortisol measures in deprived urban communities. Int J Environ Res Public Health. 2013;10(9):4086-103. https://doi.org/10.3390/ijerph10094086

21. Triguero-Mas M, Dadvand P, Cirach M, Martínez D, Medina A, Mompart A, et al. Natural outdoor environments and mental and physical health: relationships and mechanisms. Environ Int. 2015;77:35-41. https://doi.org/10.1016/j.envint.2015.01.012

22. Nayyar D, Hwang SW. Cardiovascular health issues in inner city populations. Can J Cardiol. 2015;31(9):1130-8. https://doi.org/10.1016/j.cjca.2015.04.011

23. Mitchell R, Popham F. Effect of exposure to natural environment on health inequalities: an observational population study. Lancet. 2008;372(9650):1655-60. https://doi.org/10.1016/S0140-6736(08)61689-X

24. Franchini M, Mannucci PM. Air pollution and cardiovascular disease. Thromb Res. 2012;129(3):230-4. https://doi.org/10.1016/j.thromres.2011.10.030

25. Diez-Roux AV. Residential environments and cardiovascular risk. J Urban Health. 2003;80(4):569-89. https://doi.org/10.1093/jurban/jtg065

Contribuição dos Autores: Concepção e planejamento do estudo: IHS, WLJ. Coleta, análise e interpretação dos dados: IHS. Elaboração do manuscrito: IHS. Revisão e aprovação da versão final: IHS, WLJ. Todos os autores aprovaram a versão final do manuscrito, sendo responsáveis por todos os aspectos do trabalho e garantindo sua precisão e integridade.

Conflito de Interesses: Os autores declaram não haver conflito de interesses. 